\title{
Problems of Distance Geometry and Convex Properties of Quadratic Maps*
}

\author{
A. I. Barvinok \\ Department of Mathematics, University of Michigan, \\ Ann Arbor, MI 48109-1003, USA \\ barvinok@math.lsa.umich.edu
}

\begin{abstract}
A weighted graph is called $d$-realizable if its vertices can be chosen in $d$-dimensional Euclidean space so that the Euclidean distance between every pair of adjacent vertices is equal to the prescribed weight. We prove that if a weighted graph with $k$ edges is $d$-realizable for some $d$, then it is $d$-realizable for $d=[(\sqrt{8 k+1}-1) / 2]$ (this bound is sharp in the worst case). We prove that for a graph $G$ with $n$ vertices and $k$ edges and for a dimension $d$ the image of the so-called rigidity map $\mathbb{R}^{d n} \rightarrow \mathbb{R}^{k}$ is a convex set in $\mathbb{R}^{k}$ provided $d \geq$ $[(\sqrt{8 k+1}-1) / 2]$. These results are obtained as corollaries of a general convexity theorem for quadratic maps which also extends the Toeplitz-Hausdorff theorem. The main ingredients of the proof are the duality for linear programming in the space of quadratic forms and the "corank formula" for the strata of singular quadratic forms.
\end{abstract}

\section{Introduction. Main Results}

This paper is motivated by the following problem of distance geometry. Suppose that $G=(V, E ; \rho)$ is a graph with the set of vertices $V$, the set of edges $E$, and nonnegative weights $\left\{\rho_{e}: e \in E\right\}$ on its edges. We are interested in whether it is possible to place the vertices of $G$ in the Euclidean space $\mathbb{R}^{d}$ of a given dimension $d$ so that the Euclidean distance between every pair of adjacent vertices $v_{i}, v_{j}$ would be equal to the prescribed weight $\rho_{i j}$. If a map $\varphi: V \rightarrow \mathbb{R}^{d}$ exists such that

* This research was supported by the United States Army Research Office through the Army Center of Excellence for Symbolic Methods in Algorithmic Mathematics (ACSyAM), Mathematical Sciences Institute of Cornell University, Contract DAAL03-91-C0027. 
$\| \varphi\left(v_{i}\right)-\left(\varphi\left(v_{j}\right) \|=\rho_{i j}\right.$ for every pair of adjacent vertices $v_{i}, v_{j}$ in $G$, we say that the weighted graph $G$ is $d$-realizable and call the map $\varphi$ its realization. If a graph is $d$-realizable for some $d$, then we say that the graph is realizable. The problem of $d$-realizability naturally arises in chemistry ("molecules"), mechanics ("bar and joint constructions"), robotics, and is interesting by itself (for these and related problems see, for example, [5], [7], and [11]). To test $d$-realizability of a given graph one has to test feasibility of a system of real quadratic equations. Let us denote by $\langle\cdot, \cdot\rangle$ the standard inner product in $\mathbb{R}^{d}$. A weighted graph $G=(V, E ; \rho)$ with $n=|V|$ vertices is $d$-realizable if and only if the following system of equations

(1.1) $\left\langle v_{i}, v_{i}\right\rangle+\left\langle v_{j}, v_{j}\right\rangle-2 \cdot\left\langle v_{i}, v_{j}\right\rangle=\rho_{i j}^{2} \quad$ for adjacent vertices $v_{i}$ and $v_{j}$

has a solution $v_{1}, \ldots, v_{n} \in \mathbb{R}^{d}$.

In this paper we apply some convexity arguments to deal with such systems. Equations (1.1) suggest the following notion.

(1.2) Vectorial Quadratic Equations. Let us denote by $\mathbb{R}^{n \times d}$ the linear space of all $n$-tuples $v=\left(v_{1}, \ldots, v_{n}\right)$ of $d$-vectors $v_{j} \in \mathbb{R}^{d}$. Thus $\mathbb{R}^{n \times d}$ can be identified with Euclidean $n d$-dimensional space $\mathbb{R}^{n d}$. In particular, we assume that the inner product, the norm, and the topology in $\mathbb{R}^{n \times d}$ coincide with those in $\mathbb{R}^{n d}$. Suppose that $Q=\left(q_{i j}\right), 1 \leq i, j \leq n$, is a symmetric $n \times n$ matrix. For a given $n$-tuple of $d$-vectors $v=\left(v_{1}, \ldots, v_{n}\right) \in \mathbb{R}^{n \times d}$ we define the value of the vectorial quadratic form $q$ as follows:

$$
q(v)=\sum_{i=1}^{n} q_{i i} \cdot\left\langle v_{i}, v_{i}\right\rangle+2 \cdot \sum_{1 \leq i<j \leq n} q_{i j} \cdot\left\langle v_{i}, v_{j}\right\rangle
$$

where $\langle\cdot, \cdot\rangle$ is the inner product in $\mathbb{R}^{d}$. If we consider $q$ as a real quadratic form on the Euclidean space $\mathbb{R}^{n d}$, then in a suitable basis its matrix consists of $d$ diagonal blocks, each of which is represented by the $n \times n$ matrix $Q$. For a number $d \in \mathbb{N}$, for $k$ vectorial quadratic forms $q_{1}, \ldots, q_{k}: \mathbb{R}^{n \times d} \rightarrow \mathbb{R}$, and for $k$ real numbers $a_{1}, \ldots, a_{k}$ we consider the system

$$
q_{i}\left(v_{1}, \ldots, v_{n}\right)=a_{i}, \quad v_{j} \in \mathbb{R}^{d}, \quad a_{i} \in \mathbb{R}, \quad i=1, \ldots, k, \quad j=1, \ldots, n,
$$

of vectorial quadratic equations. The larger $d$ is, the more "freedom" there is.

Thus we observe that the system (1.1) is a particular example of a system of vectorial quadratic equations (1.2.1). On the other hand, by an appropriate system of vectorial quadratic equations we can encode other geometric properties of an embedded graph in $\mathbb{R}^{d}$.

In this paper we prove the following main result.

(1.3) Theorem. If for some dimension $d \in \mathbb{N}$ a solution $v_{1}, \ldots, v_{n} \in \mathbb{R}^{d}$ to a system (1.2.1) of $k$ vectorial quadratic equations exists, then a solution to this system exists 
already for

$$
d=\left[\frac{\sqrt{8 \cdot k+1}-1}{2}\right]
$$

where $[\cdot]$ denotes the integer part.

An equivalent way to write the above bound is to define $d$ as the smallest positive integer satisfying $(d+1)(d+2) / 2>k$. We note that the bound for $d$ is independent on $n$. An immediate consequence of Theorem 1.3 is the following results.

(1.4) Corollary. Suppose that a weighted graph $G=(V, E, \rho)$ with $k=|E|$ edges is realizable. Then this graph is d-realizable for

$$
d=\left[\frac{\sqrt{8 \cdot k+1}-1}{2}\right]
$$

For example, a hexagon with nine fixed lengths of its six sides and three main diagonals is realizable if and only if it is 3-realizable. A hexagon with fixed lengths of its six sides and fixed angles between three pairs of opposite sides is realizable if and only if it is 3-realizable (since these conditions can be expressed by a system of nine vectorial quadratic equations).

It can be observed that the bound of Corollary 1.4 and thus the bound of Theorem 1.3 is sharp in the worst case. Indeed, the complete graph with $n$ vertices (that is, the 1-skeleton of an $(n-1)$-dimensional simplex) and sufficiently generic weights on its $k=n \cdot(n-1) / 2$ edges is $d$-realizable only for $d \geq n-1$, if it is realizable at all.

Theorem 1.3 is closely related to the famous Toeplitz-Hausdorff theorem (see, for example, [10], [8], and [13]) which asserts that the image of the unit sphere under certain quadratic maps is convex. Indeed, we will see that our approach leads to the following result.

(1.5) Theorem. Let $q_{1}, \ldots, q_{k}$ be vectorial quadratic forms on $\mathbb{R}^{n \times d}$. Then the image $\mathbf{q}(\mathbf{B})$ of the unit ball $\mathbf{B} \subset \mathbb{R}^{n \times d}$ under the quadratic map $\mathbf{q}: \mathbb{R}^{n \times d} \rightarrow \mathbb{R}^{k}, \mathbf{q}(v)=$ $\left(q_{1}(v), \ldots, q_{k}(v)\right)$, is convex provided

$$
d \geq\left[\frac{\sqrt{8 \cdot k+1}-1}{2}\right] \text { or, equivalently, } \frac{(d+1)(d+2)}{2}>k
$$

Varying $d$ and $k$ we obtain many convexity results similar to the Toeplitz-Hausdorff theorem.

(1.6) Corollary. The image $\mathbf{q}(B)$ of the unit ball $\mathbf{B} \subset \mathbb{R}^{n}$ under a quadratic map $\mathbf{q}: \mathbb{R}^{n} \rightarrow \mathbb{R}^{2}$ is convex. 
Proof. We put $d=1$ and $k=2$ in Theorem 1.5 .

We note that a version of the Toeplitz-Hausdorff theorem states that already the image of the unit sphere in $\mathbb{R}^{n}$ under a quadratic map $\mathbf{q}: \mathbb{R}^{n} \rightarrow \mathbb{R}^{2}$ is convex provided $n>2$ (see [10] and [13]).

(1.7) Corollary. Let $\mathbb{C}^{n}$ be a complex vector space and let $q_{1}, \ldots, q_{5}: \mathbb{C}^{n} \rightarrow \mathbb{R}$ be Hermitian forms with real coefficients. The image $\mathbf{q}(\mathbf{B})$ of the unit ball $\mathbf{B}=$ $\left\{\left(z_{1}, \ldots, z_{n}\right): \bar{z}_{1} \cdot z_{1}+\cdots+\bar{z}_{n} \cdot z_{n} \leq 1\right\}$ under a map $\mathbf{q}: \mathbb{C}^{n} \rightarrow \mathbb{R}^{5}, \mathbf{q}=\left(q_{1}, \ldots, q_{5}\right)$, is convex.

Proof. Identifying $\mathbb{C}^{n}$ with $\mathbb{R}^{n \times 2}$ we reduce Corollary 1.7 to Theorem 1.5 with $d=2$ and $k=5$.

We note that the Toeplitz-Hausdorff theorem states that the image of the unit sphere in $\mathbb{C}^{\mathbf{n}}$ under a quadratic map q: $\mathbb{C}^{\mathbf{n}} \rightarrow \mathbb{C}$ is convex provided $n>1$. A version of this theorem states that the image in $\mathbb{R}^{3}$ of the unit sphere in $\mathbb{C}^{n}$ under a map defined by three Hermitian forms is convex (see [8] and [10]).

Finally, we come back to the problem of the realizability of graphs. Let $G=$ $(V, E)$ be a graph with $n=|V|$ vertices $\{1,2, \ldots, n\}$ and $k$ edges. For every dimension $d \in \mathbb{N}$ graph $G$ defines a so-called rigidity map $\varphi_{\mathrm{G}}: \mathbb{R}^{n \times d} \rightarrow \mathbb{R}^{k}$ by the formula

$$
\varphi_{G}\left(v_{1}, \ldots, v_{n}\right)=\left(\ldots,\left\|v_{i}-v_{j}\right\|^{2}, \ldots\right) \quad \text { where } i \text { and } j \text { are adjacent in } G
$$

(see, for example, Section 3.6 of [5]).

(1.8) Corollary. Let us fix a graph $G=(V, E)$ with $k=|E|$ edges and a number $d \in \mathbb{N}$. If

$$
d \geq\left[\frac{\sqrt{8 \cdot k+1}-1}{2}\right] \text { or, equivalently, } \frac{(d+1)(d+2)}{2}>k,
$$

then the image $\varphi_{G}(\mathbf{B})$ of the unit ball $\mathbf{B} \subset \mathbb{R}^{n \times d}$ under the rigidity map $\varphi_{G}$ is a convex set in $\mathbb{R}^{k}$.

Proof. Follows by Theorem 1.5.

For a given graph $G=(V, E)$ and given weights $\left\{\rho_{e}: e \in E\right\}$ the problem of $d$-realizability is the problem of testing whether the vector $\left(\rho_{e}^{2}: e \in E\right)$ belongs to the image of the rigidity map. Corollary 1.8 implies that if $d \geq[(\sqrt{8 \cdot k+1}-1) / 2]$, then we actually have a convex problem.

\section{The Idea of the Proof. Semidefinite Programming}

In this section we present the main construction which we use to prove our results. Let us consider the linear space $W_{n}$ of all $n \times n$ real symmetric matrices. We can identify $W_{n}$ with the space $\mathbb{R}^{(n(n+1)) / 2}$. The space $W_{n}$ is equipped with the (standard) 
inner product

$$
\langle A, B\rangle=\operatorname{Tr} A B
$$

for matrices $A, B \in W_{n}$. If $A=\left(a_{i j}\right), B=\left(b_{i j}\right), 1 \leq i, j \leq n$, then

$$
\langle A, B\rangle=\sum_{i, j} a_{i j} \cdot b_{i j}
$$

We use the same notation for inner products in different spaces but this should not cause any difficulty since it is clear from the context which space is considered. We observe that this inner product is invariant under the action of the orthogonal group by conjugation, that is,

$$
\langle A, B\rangle=\left\langle G^{\mathrm{t}} A G, G^{\mathrm{t}} B G\right\rangle
$$

for an orthogonal matrix $G$ (since $G$ is orthogonal, the transposed matrix $G^{\mathrm{t}}$ coincides with the inverse matrix $G^{-1}$ ). For a matrix $X \in W_{n}$ we write $X \geq 0$ if and only if $X$ is a positive semidefinite matrix. We recall that the set of all positive semidefinite matrices in $W_{n}$ is a closed convex self-conjugate cone, that is $\langle X, Y\rangle \geq 0$ for all $Y \geq 0$ if and only if $X \geq 0$.

Suppose that $Q$ is an $n \times n$ symmetric matrix and $q$ is the corresponding vectorial quadratic form defined on $\mathbb{R}^{n \times d}(\operatorname{see}(1.2))$. For an $n$-tuple $v=\left(v_{1}, \ldots, v_{n}\right)$, $v_{i} \in \mathbb{R}^{d}$, let us define an $n \times n$ symmetric matrix $X=\left(x_{i j}\right)$ by the formula $x_{i j}=$ $\left\langle v_{i}, v_{j}\right\rangle, 1 \leq i, j \leq n$. Then (see (1.2))

$$
\begin{aligned}
q(v) & =\sum_{i=1}^{n} q_{i i}\left\langle v_{i}, v_{i}\right\rangle+2 \cdot \sum_{1 \leq i<j \leq n} q_{i j}\left\langle v_{i}, v_{j}\right\rangle \\
& =\sum_{i=1}^{n} q_{i i} x_{i i}+2 \cdot \sum_{1 \leq i<j \leq n} q_{i j} x_{i j}=\langle Q, X\rangle .
\end{aligned}
$$

We begin with the following simple result.

(2.1) Lemma. Let $q_{1}, \ldots, q_{k}: \mathbb{R}^{n \times d} \rightarrow \mathbb{R}$ be vectorial quadratic forms with the matrices $Q_{1}, \ldots, Q_{k} \in W_{n}$ and let $a_{1}, \ldots, a_{k}$ be real numbers. $A$ solution $v_{1}, \ldots, v_{n} \in \mathbb{R}^{d}$ to the system of vectorial quadratic equations

$$
q_{i}\left(v_{1}, \ldots, v_{n}\right)=a_{i}, \quad i=1, \ldots, k,
$$

exists if and only if a positive semidefinite symmetric matrix $X$ exists such that $\left\langle Q_{i}, X\right\rangle=a_{i}$ for $i=1, \ldots, k$ and rank $X \leq d$.

Proof. Suppose that $v=\left(v_{1}, \ldots, v_{n}\right), v_{j} \in \mathbb{R}^{d}$, is a solution to the above system. Let us define an $n \times n$ matrix $X=\left(x_{i j}\right)$ by the formula $X_{i j}=\left\langle v_{i}, v_{j}\right\rangle$ for $i$, $j=1, \ldots, n$. Then $q_{i}(v)=\left\langle Q_{i}, X\right\rangle$ and the matrix $X$ satisfies the desired conditions. Conversely, suppose that $X$ satisfies the conditions. Since $X$ is positive semidefinite and rank $X \leq d$ there are $n$ vectors $v_{1}, \ldots, v_{n} \in \mathbb{R}^{d}$ such that 
$x_{i j}=\left\langle v_{i}, v_{j}\right\rangle$ for $i, j=1, \ldots, n$. Then the $n$-tuple $v=\left(v_{1}, \ldots, v_{n}\right)$ is a solution to the system.

Lemma 2.1 allows us to reformulate Theorem 1.3 in the following way.

(2.2) Reformulation of Theorem 1.3. Suppose that $Q_{1}, \ldots, Q_{k}$ are $n \times n$ symmetric matrices and $a_{1}, \ldots, a_{k}$ are real numbers. If a positive semidefinite matrix $X$ exists such that

$$
\left\langle Q_{i}, X\right\rangle=a_{i}, \quad i=1, \ldots, k,
$$

then a positive semidefinite matrix $X^{*}$ exists such that it satisfies (2.2.1) and additionally

$$
\operatorname{rank} X^{*} \leq\left[\frac{\sqrt{8 \cdot k+1}-1}{2}\right]
$$

Let us consider the set of $\mathscr{X}$ of all positive semidefinite matrices $X$ such that $\left\langle Q_{i}, X\right\rangle=a_{i}, i=1, \ldots, k$. This is a closed convex set and we will find a matrix $X^{*} \in \mathscr{X}$ of the desired rank as an extreme point of $\mathscr{X}$, more precisely, as a solution to a certain optimization problem.

(2.3) Linear Programming in the Space of Quadratic Forms. For given matrices $Q_{1}, \ldots, Q_{k} \in W_{n}$, for given positive definite matrix $F \in W_{n}$, and for given real vector $a=\left(a_{1}, \ldots, a_{k}\right) \in \mathbb{R}^{k}$ let us consider the following two linear programs in $W_{n}$ :

$$
\begin{aligned}
& \text { Find } \tau=\inf \langle F, X\rangle \\
& \text { Subject to }\left\langle Q_{i}, X\right\rangle=a_{i} \text { for } i=1, \ldots, k \\
& \text { and } X \geq 0 X \in W_{n} . \\
& \text { Find } T=\sup \langle a, z\rangle \\
& \text { Subject to }\left(F-\sum_{i=1}^{k} z_{i} \cdot Q_{i}\right) \geq 0 \\
& \quad z=\left(z_{1}, \ldots, z_{k}\right) \in \mathbb{R}^{k} .
\end{aligned}
$$

Problems 2.3.1 and 2.3.2 are known under the general name "semidefinite programs" (see, for example, [2], [3], and [12]). We will show that in "general position" every optimal solution $X^{*}$ to Problem 2.3.1 satisfies the desired rank condition. To prove this, we use the linear programming duality between Problems 2.3.1 and 2.3.2, and, in particular, the complementary slackness condition. Most of the results presented below in this section are known (see, for example, [2], and [3]) or follow immediately from general theorems on linear programming in topological vector spaces [4], [12], but for the sake of completeness we present their proofs here. In Section 3 we discuss the general position of an affine subspace in $W_{n}$. Finally, in Section 4 we prove Theorems 1.3 and 1.5. We begin with the following standard duality principle. 
(2.4) Lemma. We have $\tau \geq T$. If $\tau<+\infty$ (thus a feasible point $X$ exists in Problem 2.3.1), then $\tau=T$ and the infimum in (2.3.1) is attained.

Proof. It is immediate that $\tau \geq T$. Indeed, let $X$ be a feasible point for (2.3.1) and let $z$ be a feasible point for (2.3.2). Then

$$
\left\langle F-\sum_{i=1}^{k} z_{i} \cdot Q_{i}, X\right\rangle \geq 0 \text { and therefore }\langle F, X\rangle \geq\langle a, z\rangle \text {. }
$$

We have to check that $T \geq \tau$.

Suppose that $\tau<+\infty$. It is clear that $\tau \geq 0$. Let us consider the following cone $K \subset \mathbb{R}^{k+1}$ :

$$
\begin{array}{r}
K=\left\{\left(b_{1}, \ldots, b_{k}, t\right):\left\langle Q_{i}, X\right\rangle=b_{i} \text { for } i=1, \ldots, k\right. \text { and } \\
\langle F, X\rangle=t \text { for some } X \geq 0\} .
\end{array}
$$

In other words, $K$ is the image of the cone of positive semidefinite matrices $X \in W_{n}$ under the linear map $\varphi: X \mapsto\left(\left\langle Q_{1}, X\right\rangle, \ldots,\left\langle Q_{k}, X\right\rangle,\langle F, X\rangle\right)$. Thus $K$ is a convex cone. Since $F$ is positive definite, the compact set $\{\varphi(X): X \geq 0$ and $\operatorname{Tr} X=1\}$ does not contain the origin and constitutes a compact base of $K$. Therefore, $K$ is closed. The intersection of the straight line $\Pi \subset \mathbb{R}^{k+1}, \Pi=\{(a, u): u \in \mathbb{R}\}$, with the cone $K$ is a nonempty closed interval of the type $[(a, \tau),(a, b)]$, where $b \geq \tau$ (it may happen that $b=+\infty)$. Thus the infimum in (2.3.1) is attained. Let us choose an arbitrary number $\varepsilon>0$. The point $p=(a, \tau-\varepsilon)$ does not belong to the cone $K$ and since $K$ is closed a hyperplane exists which strictly separates $p$ from $K$. Thus a vector $\left(z_{1}, \ldots, z_{k}, w\right)$ exists such that

$$
\begin{aligned}
\left\langle w \cdot F-\sum_{i=1}^{k} z_{i} \cdot Q_{i}, X\right\rangle \geq 0 & \text { for all } X \geq 0 \\
w \cdot(\tau-\varepsilon)-\langle a, z\rangle<0 & \text { for } z=\left(z_{1}, \ldots, z_{k}\right) .
\end{aligned}
$$

From the first inequality we deduce that

$$
\left(w \cdot F-\sum_{i=1}^{k} z_{i} \cdot Q_{i}\right) \geq 0
$$

since the cone of all positive semidefinite matrices is self-conjugate. Therefore, since $(a, \tau) \in K$, we have that $w \cdot \tau-\langle a, z\rangle \geq 0$. Thus $w\rangle 0$ and without loss of generality we may assume that $w=1$. Then the point $z$ is feasible for Problem 2.3.2 and $\langle a, z\rangle>\tau-\varepsilon$. Thus $T>\tau-\varepsilon$. Since we have chosen an arbitrary positive $\varepsilon$ we have that $T \geq \tau$.

The following result is the "complementary slackness" condition for Problems 2.3.1 and 2.3.2. 
(2.5) Lemma. Suppose that $z^{*}=\left(z_{1}^{*}, \ldots, z_{k}^{*}\right)$ is an optimal solution to Problem 2.3 .2 such that

$$
\operatorname{rank}\left(F-\sum_{i=1}^{k} z_{i}^{*} \cdot Q_{i}\right) \geq n-d
$$

If the set of feasible $X$ for Problem 2.3.1 is nonempty, then an optimal solution $X^{*}$ to Problem 2.3.1 exists and for any such solution we have

$$
\text { rank } X^{*} \leq d
$$

Proof. Lemma 2.4 implies that a solution $X^{*}$ exists. For such a solution $X^{*}$ by Lemma 2.4 we have

$$
\left\langle F-\sum_{i=1}^{k} z_{i}^{*} \cdot Q_{i}, X^{*}\right\rangle=\left\langle F, X^{*}\right\rangle-\sum_{i=1}^{k} z_{i}^{*} \cdot\left\langle Q_{i}, X^{*}\right\rangle=\tau-T=0 .
$$

Let us denote $A=\left(F-\sum_{i=1}^{k} z_{i}^{*} \cdot Q_{i}\right)$. Then $A$ and $X^{*}$ are positive semidefinite, rank $A \geq n-d$, and $\left\langle A, X^{*}\right\rangle=0$. Since $A$ is positive semidefinite, its eigenvalues are nonnegative and the number of positive eigenvalues is equal to rank $A$. Let us choose an orthogonal matrix $G$ such that $A_{G}=G^{-1} A G$ is a diagonal matrix whose first $n-d$ diagonal entries are strictly positive. Since the matrix $X_{G}^{*}=G^{-1} X^{*} G$ is positive semidefinite the diagonal elements of $X_{G}^{*}$ are nonnegative. Since the inner product in the space $W_{n}$ is invariant under the action of the orthogonal group by conjugation we have $\left\langle X^{*}, A\right\rangle=\left\langle X_{G}^{*}, A_{G}\right\rangle=0$, and, therefore, we must have the first $n-d$ diagonal elements of the matrix $X_{G}^{*}$ equal to zero. Again, since $X_{G}^{*}$ is positive semidefinite, we must have that all the elements in the first $n-d$ rank $X_{G}^{*} \leq d$, and, therefore, rank $X^{*} \leq d$.

We need the following technical results.

(2.6) Lemma. Suppose that $T<+\infty$ in Problem 2.3 .2 but the supremum is not attained. Then a nonzero vector $w=\left(w_{1}, \ldots, w_{k}\right) \in \mathbb{R}^{k}$ exists such that $\langle a, w\rangle=0$ and $-\sum_{i=1}^{k} w_{i} \cdot Q_{i} \geq 0$.

Proof. A sequence $z(s)=\left(z_{1}(s), \ldots, z_{k}(s)\right) \in \mathbb{R}^{k}, s=1,2, \ldots$, exists such that $\langle a$, $z(s)\rangle \rightarrow T$ as $s \rightarrow \infty$ and

$$
\left(F-\sum_{i=1}^{k} z_{i}(s) \cdot Q_{i}\right) \geq 0
$$

for all $s \in \mathbb{N}$. We must have that $\|z(s)\| \rightarrow \infty$ as $s \rightarrow \infty$ since otherwise the supremum in (2.3.2) would be attained in a limit point of the set $\{z(s): s \in \mathbb{N}\}$. Let us consider the sequence $w(s)=z(s) /\|z(s)\|$ of points on the unit sphere. This sequence has a nonzero limit point $w$ where the conditions of our lemma are satisfied. 
(2.7) Lemma. Suppose that the matrices $Q_{1}, \ldots, Q_{k}$ are linearly independent and that a positive definite matrix $X$ exists such that $\left\langle X, Q_{i}\right\rangle=a_{i}$ for $i=1, \ldots, k$. Then Problem 2.3.2 has an optimal solution.

Proof. By Lemma 2.4 we deduce that $T<+\infty$ in (2.3.2). Suppose that Problem 2.3.2 does not have an optimal solution. Let $w=\left(w_{1}, \ldots, w_{k}\right)$ be a vector from Lemma 2.6. Then

$$
\left\langle\sum_{i=1}^{k} w_{i} \cdot Q_{i}, X\right\rangle=\sum_{i=1}^{k} w_{i} \cdot a_{i}=0 .
$$

Since the matrix $\sum_{i=1}^{k} w_{i} \cdot Q_{i}$ is negative semidefinite and $X$ is positive definite we must have (see also the proof of Lemma 2.5)

$$
\sum_{i=1}^{k} w_{i} \cdot Q_{i}=0
$$

and, therefore, the matrices $Q_{1}, \ldots, Q_{k}$ are linearly dependent.

Now we can prove the main result of this section.

(2.8) Lemma. Let $Q_{1}, \ldots, Q_{k}$ be $n \times n$ symmetric matrices and let $a_{1}, \ldots, a_{k}$ be real numbers. Suppose that, for a positive definite matrix $F$ and for some number $d \in \mathbb{N}$,

$$
\operatorname{rank}\left(F-\sum_{i=1}^{k} z_{i} \cdot Q_{i}\right) \geq n-d
$$

for all $z=\left(z_{1}, \ldots, z_{k}\right) \in \mathbb{R}^{k}$. Suppose further that a positive semidefinite matrix $X$ exists such that

$$
\left\langle Q_{i}, X\right\rangle=a_{i} \quad \text { for } \quad i=1, \ldots, k
$$

Then a positive semidefinite matrix $X^{*}$ exists such that

$$
\begin{gathered}
\left\langle Q_{i}, X^{*}\right\rangle=a_{i} \quad \text { for } i=1, \ldots, k, \\
\operatorname{rank} X^{*} \geq d,
\end{gathered}
$$

and

$$
\left\langle F, X^{*}\right\rangle \leq\langle F, X\rangle \text {. }
$$

Proof. Without loss of generality we assume that the matrices $Q_{1}, \ldots, Q_{k}$ are linearly independent. Let us choose an arbitrary positive definite matrix $Y$ and put $b_{i}=\left\langle Q_{i}, Y\right\rangle$ for $i=1, \ldots, k ; b=\left(b_{1}, \ldots, b_{k}\right) \in \mathbb{R}^{k}$. For $s \in[0,1]$ let us define $X(s)=(1-s) \cdot Y+s \cdot X$ and $a(s)=(1-s) \cdot b+s \cdot a$. Then by Lemma 2.7 for any $s<1$ Problem 2.3 .2 with the vector $a(s)$ has an optimal solution since the positive definite matrix $X(s)$ is a feasible point for Problem 2.3.1. Thus by Lemma 2.5 
a solution $X^{*}(s)$ of Problem 2.3.1 exists such that rank $X^{*}(s) \leq d$. Besides, we have that $\left\langle F, X^{*}(s)\right\rangle \leq s \cdot\langle F, X\rangle+(1-s) \cdot\langle F, Y\rangle$; in particular, the family $\left\{X^{*}(s): 0 \leq s<1\right\}$ is bounded. Therefore for a sequence $s_{m} \rightarrow 1$ a limit $X^{*}$ of the sequence $X^{*}\left(s_{m}\right)$ exists. The matrix $X^{*}$ satisfies conditions (2.8.1)-(2.8.3).

\section{General Position in the Space of Quadratic Forms}

In this section we recall some facts about quadratic forms "in general position." We are interested in the "corank stratification" of the space of quadratic forms $W_{n}$.

(3.1) Proposition (see, for example, the corollary of Lemma 2 from [1]). For any $r=0, \ldots, n$ the set

$$
W_{n}^{r}=\left\{A \in W_{n}: \operatorname{rank} A=n-r\right\}
$$

is a smooth analytic variety in $W_{n}$ of codimension $(r \cdot(r+1)) / 2$.

Thus $W_{n}^{n}=\{0\}$ is the origin in $W_{n}$ whereas $W_{n}^{0}$ is a dense subset in $W_{n}$. Proposition 3.1 is sometimes referred to as the "corank formula."

(3.2) Definition. Let us fix symmetric $n \times n$ matrices $Q_{1}, \ldots, Q_{k}$. We say that a symmetric $n \times n$ matrix $F$ is regular if and only if the map $\psi_{F}: \mathbb{R}^{k} \rightarrow W_{n}$ given by the formula

$$
\psi_{F}\left(z_{1}, \ldots, z_{k}\right)=F-\sum_{i=1}^{k} z_{i} \cdot Q_{i}
$$

intersects every variety $W_{n}^{r} \subset W_{n}, r=0, \ldots, n$, transversally. If it is not clear from the context we specify that $F$ is regular with respect to $Q_{1}, \ldots, Q_{k}$.

The following result justifies Definition 3.2.

(3.3) Proposition. For given $n \times n$ symmetric matrices $Q_{1}, \ldots, Q_{k}$ the set of regular matrices $F$ contains an open and dense subset of $W_{n}$.

Proof. Let us consider a linear map.

$\varphi: \mathbb{R}^{k} \oplus W_{n} \rightarrow W_{n}, \quad \varphi(z, F)=F-\sum_{i=1}^{k} z_{i} \cdot Q_{i}, \quad z=\left(z_{1}, \ldots, z_{k}\right) \in \mathbb{R}^{k}, \quad F \in W_{n}$.

The map $\varphi$ is surjective and therefore intersects every variety $W_{n}^{r}$ transversally. For $r=0, \ldots, n$ let us put $S_{r}=\varphi^{-1}\left(W_{n}^{r}\right) \subset \mathbb{R}^{k} \oplus W_{n}$. Let us denote by $p$ the natural projection $p: \mathbb{R}^{k} \oplus W_{n} \rightarrow W_{n}$ onto the second summand. It then follows (see, for example, Lemma 4.6 of [9]) that $F$ is regular if it is a regular value of every restriction $p_{r}: S_{r} \rightarrow W_{n}$ of $p$ on $S_{r}$. Since the set of singular values of $p_{r}$ is a semialgebraic set, by Sard's lemma (see [9]) we get the desired result.

Below we prove the main result of this section. 
(3.4) Corollary. Suppose that $Q_{1}, \ldots, Q_{k}$ are real symmetric $n \times n$ matrices. Then for a regular symmetric $n \times n$ matrix $F$ and for

$$
d=\left[\frac{\sqrt{8 \cdot k+1}-1}{2}\right]
$$

where $[\cdot]$ denotes the integer part, we have that

$$
\operatorname{rank}\left(F-\sum_{i=1}^{k} z_{i} \cdot Q_{i}\right) \geq n-d
$$

for all $z=\left(z_{1}, \ldots, z_{k}\right) \in \mathbb{R}^{k}$.

Proof. Let us consider the following affine subspace $\mathscr{A}$ in the space $W_{n}$ :

$$
\mathscr{A}=\left\{F-\sum_{i=1}^{k} z_{i} \cdot Q_{i}: z_{1}, \ldots, z_{k} \in \mathbb{R}\right\} .
$$

Then the dimension of $\mathscr{A}$ does not exceed $k$ and by Proposition 3.1 and Definition 3.2 it follows that $\mathscr{A}$ does not intersect the set $W_{n}^{r}$ provided $r \cdot(r+1) / 2>k$. Hence for $r \cdot(r+1) / 2>k$ we have that

$$
\operatorname{rank}\left(F-\sum_{i=1}^{k} z_{i} \cdot Q_{i}\right) \geq n-r+1
$$

for all $z_{1}, \ldots, z_{k} \in \mathbb{R}$. We choose $r=d+1$ and the proof follows.

\section{Proofs and Final Remarks}

Proof of Theorem 1.3. We prove the Reformulation of Theorem 1.3 (see (2.2)). By Proposition 3.3 we can choose a positive definite matrix $F$ which is regular with respect to $Q_{1}, \ldots, Q_{k}$. Then, by Corollary 3.4 for all $z=\left(z_{1}, \ldots, z_{k}\right) \in \mathbb{R}^{k}$,

$$
\operatorname{rank}\left(F-\sum_{i=1}^{k} z_{i} \cdot Q_{i}\right) \geq n-d, \quad \text { where } d=\left[\frac{\sqrt{8 \cdot k+1}-1}{2}\right] \text {. }
$$

Now we use Lemma 2.8.

Proof of Theorem 1.5. Let $Q_{1}, \ldots, Q_{k} \in W_{n}$ be the matrices of the vectorial quadratic forms $q_{1}, \ldots, q_{k}: \mathbb{R}^{n \times d} \rightarrow \mathbb{R}$. By Proposition 3.3 we can choose a positive definite matrix $F$ which is regular with respect to $Q_{1}, \ldots, Q_{k}$. Let $f: \mathbb{R}^{n \times d} \rightarrow \mathbb{R}$ be the vectorial quadratic form with the matrix $F$. First, we prove that the image $\mathbf{q}\left(\mathbf{E}_{f}\right)$ of the elipsoid

$$
\mathbf{E}_{f}=\left\{v \in \mathbb{R}^{n \times d}: f(v) \leq 1\right\}
$$


is convex. Let us prove that the image $\mathbf{q}\left(\mathbf{E}_{f}\right)$ coincides with the image of a convex set

$$
A=\left\{X \in W_{n}: X \geq 0 \text { and }\langle F, X\rangle \leq 1\right\}
$$

under a linear map

$$
\varphi: X \mapsto\left(\left\langle Q_{1}, X\right\rangle, \ldots,\left\langle Q_{k}, X\right\rangle\right) .
$$

Lemma 2.1 implies that $\mathbf{q}\left(\mathbf{E}_{f}\right) \subset \varphi(A)$. Let us prove the opposite inclusion. Indeed, let us take an arbitrary point $a=\left(a_{1}, \ldots, a_{k}\right) \in \varphi(A)$. Then a positive semidefinite matrix $X$ exists such that $\left\langle Q_{i}, X\right\rangle=a_{i}$ for $i=1, \ldots, k$ and $\langle F, X\rangle \leq 1$. Since $F$ is regular, by Corollary 3.4 and Lemma 2.8 we conclude that a positive semidefinite matrix $X^{*}$ exists such that $\left\langle Q_{i}, X^{*}\right\rangle=a_{i}$ for $i=1, \ldots, k,\left\langle F, X^{*}\right\rangle \leq 1$, and additionally rank $X^{*} \leq d$. Now by Lemma 2.1 it follows that a $v \in \mathbb{R}^{n \times d}$ exists such that $f(v) \leq 1$ and $q(v)=a$. Since the set $\varphi(A)$ is convex the image $Q\left(\mathbf{E}_{f}\right)$ is also convex.

To conclude the proof, we observe that the identity matrix can be approximated by a regular matrix with an arbitrary small error (see Proposition 3.3). This is, for any $\varepsilon>0$ a positive definite regular matrix $F$ exists such that the ellipsoid $\mathbf{E}_{f}$ approximates the unit ball $\mathbf{B} \in \mathbb{R}^{n \times d}$ within the margin less than $\varepsilon$,

$$
(1-\varepsilon) \cdot \mathbf{E}_{f} \subset \mathbf{B} \subset(1+\varepsilon) \cdot \mathbf{E}_{f} .
$$

Hence we conclude that the image $\mathbf{q}(\mathbf{B})$ is convex.

The crucial component of the above proofs is the observation that a certain affine subspace

$$
F-\sum_{i=1}^{k} z_{i} \cdot Q_{i}, \quad z_{1}, \ldots, z_{k} \in \mathbb{R},
$$

and does not intersect certain varieties $W_{n}^{r}$ of singular forms in the space of quadratic forms $W_{n}$. This allows us to improve the bound of $d$ in Theorems 1.3 and 1.5 if our matrices $Q_{1}, \ldots, Q_{k}$ have a special structure. A particular example of such a structure is given below.

(4.1) Example. $r$-Diagonal Matrices. We say that a symmetric matrix $Q=\left(q_{i j}\right)$, $1 \leq i, j \leq n$, is $r$-diagonal if and only if $q_{i j}=0$ unless $|i-j|<r$. Suppose that $Q_{1}, \ldots, Q_{k}$ are symmetric $n \times n$ r-diagonal matrices. Let us pick up an $n \times n$ symmetric positive definite matrix $F=\left(f_{i j}\right), 1 \leq i, j \leq n$, of the following type:

$$
f_{i j}= \begin{cases}1 & \text { if } i=j, \\ \varepsilon_{i j}=\varepsilon_{j i}>0 & \text { if }|i-j|=r, \\ 0 & \text { elsewhere. }\end{cases}
$$

Then, for all $z=\left(z_{1}, \ldots, z_{k}\right) \in \mathbb{R}^{k}$,

$$
\operatorname{rank}\left(F-\sum_{i=1}^{k} z_{i} \cdot Q_{i}\right) \geq n-r,
$$


since the $(n-r) \times(n-r)$ submatrix of this linear combination consisting of the $(i, j)$-entries with $i=1, \ldots, n-r$ and $j=r+1, \ldots, n$ is nondegenerate (as a lower triangular matrix with nonzero epsilons on the main diagonal). The proof of the following convexity result is completely analogous to the proof of Theorem 1.5.

(4.1.1) Proposition. Let us fix $r \in \mathbb{N}$. Suppose that $q_{1}, \ldots, q_{k}: \mathbb{R}^{n \times d} \rightarrow \mathbb{R}$ are vectorial quadratic forms whose matrices are r-diagonal. Then the image $\mathbf{q}(\mathbf{B})$ of the unit ball $\mathbf{B} \subset \mathbb{R}^{n \times d}$ under the quadratic map $\mathbf{q :} \mathbb{R}^{n \times d} \rightarrow \mathbb{R}^{k}, \mathbf{q}(v)=\left(q_{1}(v), \ldots, q_{k}(v)\right)$, is convex provided $d \geq r$.

In particular, if $d=r=1$, then $q_{1}, \ldots, q_{k}$ are diagonal forms. After the substitution $y_{i}=x_{i}^{2}, i=1, \ldots, n$, the unit ball $\mathbf{B}=\left\{x=\left(x_{1}, \ldots, x_{n}\right): x_{1}^{2}+\ldots+x_{n}^{2} \leq 1\right\}$ in $\mathbb{R}^{n}=\mathbb{R}^{n \times 1}$ transforms into the simplex $\Delta=\left\{\left(y_{1}, \ldots, y_{n}\right): y_{i} \geq 0\right.$ for $i=1, \ldots n$ and $\left.y_{1}+\cdots+y_{n} \leq 1\right\}$ whereas the quadratic forms $q_{1}(x), \ldots, q_{k}(x)$ transform into linear forms in $y_{1}, \ldots, y_{n}$. Hence we may consider Proposition 4.1.1 as a generalization of the fact that the image of a convex polytope under a linear map is convex. In particular, we get the following "complex version" of this elementary fact.

(4.1.2) Corollary. Let $\mathbb{C}^{n}$ be a complex vector space and let $q_{1}, \ldots, q_{k}: \mathbb{C}^{n} \rightarrow \mathbb{R}$ be Hermitian forms whose matrices are real and 2-diagonal. Then the image $\mathbf{q}(\mathbf{B})$ of the unit ball $\mathbf{B}=\left\{\left(z_{1}, \ldots, z_{n}\right): \bar{z}_{1} \cdot z_{1}+\cdots+\bar{z}_{n} \cdot z_{n} \leq 1\right\}$ under the map $\mathbf{q}: \mathbb{C}^{n} \rightarrow \mathbb{R}^{k}$, $\mathbf{q}=\left(q_{1}, \ldots, q_{k}\right)$, is convex.

Proof. Identifying $\mathbb{C}^{n}$ and $\mathbb{R}^{n \times 2}$ we reduce Corollary 4.1 .2 to Proposition 4.1.1 with $r=2$.

(4.2) Computational Questions. We have not discussed yet how to construct a realization in $\mathbb{R}^{d}$ of a given weighted graph even if we know that such a realization exists. If the dimension $d$ satisfies the condition of Corollary 1.4 , then such a realization can be obtained by solving the semidefinite program (2.3.1) with a regular positive definite matrix $F$. For a problem of this type either the ellipsoid method or an efficient interior point method can be applied (for computational aspects see [2], [3], and [12]).

(4.3) Possible Applications to Rigidity. Connelly suggested that Theorem 1.3 can be applied not only to the problem of realization (that is, existence of a configuration with prescribed distances) but also to the problem of rigidity (that is, local or global uniqueness of the configuration). For example, second-order rigidity can be expressed in terms of vectorial quadratic equations. It seems that our matrix $F$ plays a role similar to that of "stress" in [6].

\section{Acknowledgments}

I am grateful to A. M. Vershik for numerous fruitful discussions during our work on the project "Convex Analysis and Linear Programming." The approach to the 
Toeplitz-Hausdorff theorem described in this paper was inspired by these discussions and by [13]. I am indebted to Peter Mani who has drawn my attention to the problem of realizability of graphs and to Robert Connelly who has introduced me to the problems of rigidity. Conversations with Farid Alizadeh were very useful for me.

\section{References}

1. A. A. Agrachev, Topology of quadratic maps and Hessians of smooth maps (in Russian), Itogi Nauki i Tekhniki, Seriya Algebra, Topologiya, Geometriya, 26 (1988), 85-124, translated in Joumal of Soviet Mathematics, 49(3) (1990), 990-1013.

2. F. Alizadeh, Optimization over positive semi-definite cone; interior-point methods and combinatorial applications, in: Advances in Optimization and Parallel Computing, $\mathbf{P}$. Pardalos, ed., North-Holland, Amsterdam, 1992, pp. 1-25.

3. F. Alizadeh, Interior point methods in semidefinite programming with applications to combinatorial optimization, to appear.

4. E. Anderson and P. Nash, Linear Programming in Infinite Dimensional Spaces, Wiley, New York, 1987.

5. R. Connelly, Rigidity, in: Handbook of Convex Geometry, P. M. Gruber and J. M. Wills, eds., Elsevier, Amsterdam, 1993, Chapter 1.7, pp. 223-271.

6. R. Connelly and W. Whitely, Second-order rigidity and pre-stress stability for tensegrity frameworks, to appear.

7. G. M. Crippen and T. F. Havel, Distance Geometry and Molecular Conformation, Wiley, New York, 1988.

8. Ch. Davis, The Toeplitz-Hausdorff theorem explained, Canadian Mathematical Bulletin, 14(2) (1971), 245-246.

9. M. Golubitsky and V. Guillemin, Stable Mappings and Their Singularities, Springer-Verlag, New York, 1973.

10. P. R. Halmos, A Hilbert Space Problem Book, Van Nostrand, London, 1967.

11. B. Jaggi, Configuration spaces of planar polygons, Preprint, 1992.

12. Yu. Nesterov and A. Nemirovskii, Interior-Point Polynomial Algorithms in Convex Programming, Studies in Applied Mathematics, Vol. 13, SLAM, Philadelphia, PA, 1993.

13. A. M. Vershik, Quadratic forms positive on a cone and quadratic duality (in Russian), Zapiski Nauchnuh Seminarov Leningradskogo Matematicheskogo Institita im. V. A. Steklova AN SSSR, 134 (1984), 59-83, translated in Journal of Soviet Mathematics, 36(1), 39-56.

Received February 16, 1994. 